DOSSIER

\title{
CÉLINE SCHMIDT
}

PHILIPPE ROBERT-DEMONTROND

IGR, IAE de Rennes

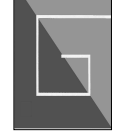

\section{Territoire et mobilité géographique infranationale}

\author{
Étude des représentations \\ et des trajectoires des salariés
}

Si la mobilité géographique des salariés est un problème managérial d'importance majeure, elle n'est reconnue comme tel que dans sa dimension internationale. Sa dimension infranationale est, quant à elle, peu questionnée. Elle n'est le plus souvent pas reconnue comme faisant problème, et n'est guère problématisée. Pour contribuer à pallier ce manque, une étude exploratoire est engagée, d'inspiration ethnosociologique. Nous pointons l'existence de vives difficultés d'adaptation et soulignons l'importance d'étudier le « capital de mobilité » des salariés. 
$\mathrm{L}$ a gestion des ressources humaines est actuellement marquée, en matière de mobilité géographique des salariés à l'infranational, par un préjugé d'absence de problèmes autres que ceux purement techniques. Nombreuses sont ainsi les entreprises qui, lors de licenciements économiques, reclassent hâtivement les salariés sur d'autres sites, quand elles ne font pas de la mobilité géographique un impératif dans la trajectoire professionnelle des individus.

Ainsi de France Télécom, typiquement, mettant en place, en 2007, un programme de mobilité nommé « Time to move », amenant les cadres à changer d'affectation tous les trois ans : cas exemplaire de cet imaginaire managérial où les salariés sont présupposés sans attaches. Imaginaire de fluidité, de liquidité, qui domine les esprits de manière telle que l'attachement territorial est un impensé et un impensable.

Cette conception d'un rapport « lisse » au territoire pose des problèmes inattendus en France : la mobilité géographique n'est en vérité pas d'évidence, à l'échelle infranationale même. Elle peut générer, non seulement lorsqu'elle est imposée, mais encore lorsqu'elle est voulue, des souffrances d'importance chez les salariés.

Nous rapportons dans cet article une étude empirique, de type ethnosociologique, qui met en lumière les difficultés d'adaptation des salariés en situation de mobilité infranationale. Nous présentons ainsi une réflexion critique du cadre conceptuel actuellement mis en avant en gestion des ressources humaines, focalisé sur l'aspect cognitif des problèmes d'adaptation. Nous proposons un changement de paradigme, intégrant les aspects expérientiels et émotionnels de la mobilité géographique. Nous illustrons enfin de diverses données empiriques les difficultés qui, pouvant être vécues par les salariés lors de mobilités à l'infranational, témoignent de l'importance des attaches territoriales.

\section{I - IMOBILITÉ GÉOGRAPHIQUE OU ATTACHEMENT AU TERRITOIRE ?}

\section{La mobilité géographique en management}

Selon l'un des principaux arguments en faveur d'une plus grande mobilité géographique en France, la rationalisation de la production « exige une plus grande flexibilité, adaptabilité et disponibilité de la part des salariés » (Vignal, 2006, p. 102). Toutes qualités valorisées qui impliquent leur parfaite mobilité, sans attaches territoriales. Cette axiologie incline à une ontologie, à une anthropologie silencieuse. L'homme est conçu naturellement mobile ; son aptitude au nomadisme, interprétée comme une indifférence affective aux territoires, est une évidence, pour la doxa managériale, qui ne perçoit pas l'espace comme une contrainte, comme un problème potentiel.

Les travaux managériaux s'intéressant à la mobilité géographique traitent essentiellement celle-ci dans une perspective cognitiviste (Robert-Demontrond, 2001), insistant sur des problèmes d'information, de traitement d'information, et portant une vision utilitariste - l'individu est conçu tel qu'effectuant ses choix de mobilité en fonction d'un arbitrage coûts/bénéfices. L'incertitude de l'environnement est postulée induire, chez les salariés, des perturbations de routines - celles-ci étant plus importantes en mobilité internationale qu'en 
mobilité nationale. S'appuyant sur les travaux d'Oberg (1960), la prévention des problèmes est cadrée par des programmes visant essentiellement à diminuer cette incertitude. Les préconisations managériales portent essentiellement sur: 1) la sélection des salariés, grâce à la détection de caractéristiques individuelles susceptibles d'influencer la disposition envers la mobilité ; 2) la formation des salariés, qui consiste en programmes d'apprentissage socioculturel permettant au salarié de développer des compétences relationnelles; 3) la prise en compte de la famille dans le processus décisionnel, avec un soutien informationnel, mais aussi matériel et financier.

Trop peu de travaux s'intéressent à la mobilité géographique à l'échelle nationale (pour une synthèse, voir Guillot-Soulez et Sergot, 2010). L'analyse porte essentiellement, dans le cas français sur : i) la mobilité interne et intra-organisationnelle lors de transferts d'établissements, ou de restructurations d'entreprise; ii) les variables favorisant ou limitant la mobilité ; iii) une population homogène de salariés, les mobiles - hauts cadres (Mignonac, 2001), militaires ou encore étudiants -, et les non mobiles - les résistants au changement - ; iv) les cas de mobilité « collective », contrainte par des changements organisationnels (Roger et Roques, 2004). Si certains travaux sociologiques tentent d'insérer des variables relationnelles dans le modèle décisionnel, comme « la communauté actuelle », « l'attachement territorial familial » (Vignal, 2005), ces études font exclusivement référence, d'une part, à des formes particulières de mobilité et d'autre part, à un certain type de liens sociaux, excluant finalement les liens au territoire (à l'environnement physique). Les solutions envisagées sont essentiellement tournées vers la communication, l'apprentissage cognitif et le développement de compétences.

$\mathrm{Au}$ total, la recherche en sciences de gestion, et en ressources humaines en particulier, n'épuise pas toutes les formes de mobilité géographique, ni tous les phénomènes liés à celle-ci et, ne s'interroge pas suffisamment en profondeur sur le sens que les salariés donnent à ce type d'expérience, notablement lorsqu'elle correspond à des déplacements à une échelle infranationale.

\section{Du territoire à l'attachement au territoire}

La gestion de la mobilité géographique des salariés est souvent étudiée sous l'angle exclusif du lien entre le salarié et son entreprise (ici comprise comme un lieu de travail). Pour comprendre les difficultés d'adaptation vécues suite à tout type de mouvement géographique, nous notons l'absence de prise en compte : i) des autres lieux ; ii) des mécanismes de l'attachement au territoire; et iii) de ses effets.

Le territoire est non seulement «une dimension oubliée du management stratégique », comme le constatent Lauriol et al. (2008), il est aussi une dimension oubliée du management de la mobilité géographique des salariés. Pour l'étudier, nous choisissons ici une approche subjective, originale, à notre connaissance encore jamais utilisée en management, en retenant pour définition du territoire l'idée d'un « espace vécu, (qui) exprime le rapport existentiel, forcément subjectif, que l'individu socialisé (donc informé et influencé par la sphère 
sociale) établit avec la Terre. Il s'imprègne de valeurs culturelles reflétant, pour chacun, l'appartenance à un groupe localisé. Sa connaissance passe par l'écoute des acteurs, par la prise en compte de leurs pratiques et de leurs imaginaires spatiaux » (Di Méo, 1998). Une telle approche phénoménologique met l'accent sur: i) l'incorporation du corps dans l'espace; ii) l'aspect ressource du territoire ; iii) le lien entre le territoire et l'identité. Nous reprenons les différentes échelles de territoire identifiées par Ravelli (2008) pour différencier les mobilités locale (départementale), régionale, interrégionale, selon les successions de lieux de vie. La définition de l'attachement au territoire n'étant pas stabilisée, nous avons regroupés plusieurs définitions, afin de proposer celle-ci : un lien affectif, cognitif, identitaire entre une personne et tout type de lieu - lien résultant d'une dynamique existentielle.

En définitive, il ressort de l'examen de la littérature actuellement développée sur la mobilité géographique que les études effectuées sont majoritairement conduites selon une conception cognitiviste du rapport de l'homme au changement de lieux, de milieux. Les modèles qui en découlent sont explicatifs, à visée nomologique. D'autres perspectives sont possibles et restent à découvrir - perspectives phénoménologiques, en lien avec les travaux sur l'attachement au territoire. D'autres méthodologies que celle, fortement quantitative, actuellement dominante sont envisageables - investigations qui appellent une herméneutique du vécu de la mobilité géographique, à travers la prise en compte du territoire comme espace investi d'affects (Di Méo, 1998). Ce que nous retenons ici.

\section{II - MOBILITÉ INFRANATIONALE ET ATTACHEMENT AU TERRITOIRE : MÉTHODOLOGIE ET RÉSULTATS}

\section{Choix méthodologiques}

Compte tenu de la sensibilité et de la complexité des phénomènes à l'étude, la démarche choisie s'inscrit dans le champ méthodologique de l'ethnosociologie, afin de saisir le vécu des salariés dans ses dimensions affectives outre celles cognitives. L'approche ainsi suivie permet la combinaison de plusieurs méthodes, entre lesquelles nous avons choisi celle dite des « récits de vie», comme principale voie d'investigation. Cette technique d'investigation mobilise une forme particulière d'entretien intersubjectif, l'entretien narratif, au cours duquel est demandé à une personne (reconnue comme sujet) de "raconter tout ou partie de son expérience vécue » (Bertaux, 2005, p. 6). Le recours aux entretiens narratifs donne ainsi aux chercheurs l'opportunité de saisir le vécu dans toute sa complexité, cognitive et affective, de comprendre une trajectoire existentielle, de saisir, ici, les représentations spatiales et temporelles des salariés. Avec d'autant plus de pertinence que le récit permet une investigation non précadrée par des attentes. Pour la construction de l'échantillon, nous avons retenu toute personne ayant vécu, ou vivant au moment de l'enquête, une mobilité géographique. Nous entendons par là, «le changement d'une aire géographique vers une autre, par exemple, région, département, bassin d'emploi " (Lemoine et Wasmer, 2010, p. 19). Le concept de mobilité résidentielle (Vignal, 2005) n'est pas satisfaisant, puisqu'il ne prend pas en compte les personnes qui ont une double résidence 
(appelées encore les «couples TGV»), alors même que ces situations sont de plus en plus fréquentes aujourd'hui. L'échantillon a évolué en fonction de l'analyse des premiers entretiens et des retours à la théorie ; le nombre total d'entretiens effectués a été décidé suivant l'application des principes : i) de diversification - avoir des cas variés a priori, afin d'obtenir une saturation sémantique (Glaser et Strauss, 1967); et ii) de falsification - retenant comme objectif la recherche de « cas négatifs » (Bertaux, 2005). Dans cette perspective de construction progressive, ont été retenus comme critères de sélection : l'âge, le genre, le statut familial, la catégorie socioprofessionnelle, le secteur d'activité, la provenance géographique (dix régions de France).
Afin d'illustrer nos analyses, nous rapportons plusieurs verbatims issus du corpus textuel constitué (les caractéristiques des informateurs sont répertoriées dans le tableau 1).

\section{Principaux résultats}

Les résultats obtenus sont classables en deux catégories principales relatives : i) aux tensions qui peuvent exister dans la trajectoire du salarié (entre mobilité et immobilité, ou encore entre une situation existentielle sans attaches et avec attaches) ; ii) aux tensions apparaissant dans l'organisation, entre salariés.

\section{La complexité du processus d'adaptation}

La mobilité géographique ne signifie pas nécessairement un départ sans horizon de

\section{MÉTHODOLOGIE}

Nous avons recueilli trente-deux récits de vie, auprès de dix-neuf hommes et de treize femmes (durée moyenne : une heure et demie). La phrase introductive de l'entretien se veut très ouverte : « racontez-nous votre expérience de la mobilité géographique ». Dans une perspective de triangulation des données, nous avons croisé ce premier mode de production d'informations avec une ethnographie virtuelle (Hine, 2000). L'intérêt de cette méthode est d'ouvrir significativement le «champ spatial» de l'investigation par le suivi continu de blogs et de forums de discussion : l'importance des partages et des échanges sur le net s'explique par l'intensité émotionnelle de cette expérience. En parallèle, une "observation flottante " (Juan, 2005), a permis d'alimenter en continu la tenue d'un carnet de terrain en informations diversement collectées, au quotidien : le suivi régulier (en accompagnement), de salariés vivant une mobilité, a été couplé à la prise de notes, en impromptu, lors de discussions informelles, au hasard de rencontres imprévues. Enfin, divers documents, rapports d'enquêtes académiques et articles de journaux, ont alimenté la masse de matériaux à analyser. L'étude de données secondaires a permis de consolider et conforter l'analyse. Le traitement du matériau qualitatif ainsi constitué a été effectué suivant la méthode d'analyse manuelle et d'interprétation de données développée par Paillé et Mucchielli (2008). L'analyse de la narration (Ricœur, 1990) a été effectuée à l'aide d'un codage ouvert et d'une confrontation à la littérature - la construction des « catégories conceptualisantes » permettant la théorisation. 
Tableau 1 - Informateurs

\begin{tabular}{|c|c|}
\hline $\begin{array}{l}\text { Matériaux : récit, } \\
\text { suivi, ethnographie } \\
\text { virtuelle }\end{array}$ & Profil des informateurs \\
\hline $\mathrm{R}$ et $\mathrm{S}$ pendant 3 ans & $\begin{array}{l}\text { Stéphane : } 27 \text { ans, en couple sans enfant ; technicien dans une } \\
\text { multinationale } \\
\text { Mobilité : Lorraine vers Normandie depuis } 2007 \\
\text { Avril } 2011 \text { : rupture avec l'entreprise, dépression, séparation avec sa } \\
\text { compagne ; mai } 2011 \text { : retour en Lorraine }\end{array}$ \\
\hline $\mathrm{R}$ et $\mathrm{S}$ pendant 2 ans & $\begin{array}{l}\text { Anna : } 28 \text { ans, en couple sans enfant ; agent de la fonction publique } \\
\text { Mobilités : Marseille vers Montpellier, puis Rennes }\end{array}$ \\
\hline $\mathrm{R}$ et $\mathrm{S}$ pendant 2 ans & $\begin{array}{l}\text { Yann : } 27 \text { ans, en couple sans enfant ; agent de la fonction publique } \\
\text { Mobilités : Strasbourg vers Montpellier, puis Rennes } \\
\text { Décembre } 2010 \text { : sa compagne est affectée à Chartres } \\
\text { Janvier } 2012: \text { mi-temps annualisé, suite à plusieurs demandes de } \\
\text { mutation }\end{array}$ \\
\hline $\mathrm{R}$ & $\begin{array}{l}\text { Tania : } 24 \text { ans ; en couple sans enfant ; auxiliaire de vie } \\
\text { Mobilité : Lorraine vers Alsace }\end{array}$ \\
\hline EV & $\begin{array}{l}\text { Enna : la vingtaine; célibataire } \\
\text { Mobilité : Bretagne vers Île-de-France, } \\
\text { http://www.forumdesados.net.mal-du-pays }\end{array}$ \\
\hline EV & $\begin{array}{l}\text { Jocelin : } 26 \text { ans ; célibataire ; sportif de haut niveau } \\
\text { Mobilité : Bourgogne vers Midi-Pyrénées } \\
\text { http://www.lejsl.com/edition-d-autun }\end{array}$ \\
\hline EV & $\begin{array}{l}\text { Marie : } 30 \text { ans ; en couple avec enfant } \\
\text { Mobilité : Bretagne vers Aquitaine, depuis } 7 \text { ans } \\
\text { http://forum.aufeminin.com }\end{array}$ \\
\hline EV & $\begin{array}{l}\text { Nicolas : la quarantaine ; cadre dans la finance } \\
\text { Mobilité : Paris vers Toulouse ; http://www.toutpourchanger.com }\end{array}$ \\
\hline EV & $\begin{array}{l}\text { Léo : données non communiquées (NC) } \\
\text { http://voyageforum.com/voyage }\end{array}$ \\
\hline $\mathrm{EV}$ & Luc : NC; www.antourtan.org/events/enquete01.htm \\
\hline
\end{tabular}

Note : $\mathrm{R}$ : récit, $\mathrm{S}:$ suivi, $\mathrm{EV}:$ ethnographie virtuelle.

retour, ou avec des perspectives de retour envisagées sur un lointain futur. Parfois s'observent au contraire des retours très fréquents vers le lieu d'origine - «lieu des attaches ». Le salarié se pense alors «en transit »; il peut définir sa mobilité comme quelque espèce de simple «déplacement professionnel » - alors même que l'adaptation au travail, à l'organisation, se passe bien, il n'est en ce cas aucunement question d'implication. La vie, dans la région des investissements fonctionnels (liés au travail), est comme suspendue dans l'attente du retour (le plus fréquent possible), dans la 
région d'origine ; région de tous les investissements émotionnels. Le cas de Stéphane est exemplaire de ce type de vécu. Originaire de Lorraine, il travaille en Normandie - où il habite depuis deux ans. « Je ne me sens même pas totalement intégré... en Normandie, alors que ça fait deux ans que je suis en Normandie (...). Donc, bizarrement, même si je suis en Normandie... (...) Ma vie extra professionnelle est plus régie par la Moselle que par la Normandie (...). J'organise des retours en fonction des activités que j'ai en Moselle. Je suis en Normandie en fait c'est bizarre, c'est comme si j'étais en déplacement, tu vois, pour moi, c'est ça, c'est un peu ça, je suis en Normandie, je suis en déplacement (...) Moi, la Lorraine c'est chez moi et la Normandie, c'est l'endroit où je travaille, même si j'ai un appartement, que j'ai fait toutes les démarches administratives parce que je vis au Havre ! (...) Je ne suis pas quelqu'un qui est parti, enfin qui, tu vois... qui... a déplacé sa vie en Normandie. C'est pas quelqu'un qui est parti de la Lorraine et qui vit en Normandie, je suis quelqu'un qui a sa vie en Lorraine mais qui va travailler en Normandie. Si je pouvais rentrer tous les soirs, je rentrerais tous les soirs (...) je suis en déplacement, je suis en déplacement, c'est fou...» (Stéphane). Les difficultés économiques de la Lorraine ont contraint cet informateur au départ - vécu comme un vrai exil. La région de travail, région d'accueil, ne peut être pour lui réellement hospitalière : on ne peut y être « comme chez soi ». Il n'y a, pour lui, qu'un « chez-soi », un et un seul : sa région. Elle seule est vécue comme un territoire - les autres régions sont des terres fonctionnelles - non pas des « lieux », mais des « non-lieux », au sens d'Augé (1992), des espaces de pas- sage, sans possible attache, des espaces ni identitaires, ni relationnels. «De toute façon, moi, je suis l'exemple même du gars qui a bougé pour le travail, qui n'a bougé que pour le travail. Parce que, partir c'était pas un besoin, y'a des gens, ils ont besoin de partir, de connaître autre chose, de connaître une autre région, un autre pays. Moi, je suis parti exclusivement pour le travail » (Stéphane).

Le problème de la mobilité géographique n'est pas économique, et ne se résout pas économiquement. Il n'y a pas de compensation financière à une peine profondément existentielle. Les témoignages recueillis révèlent ainsi que, typiquement, l'aide financière accordée pour les déménagements n'empêche aucunement, une fois sur place, d'éprouver un mal-être... Parfois, ce mal-être qu'induit l'attachement territorial peut amener les salariés partis de leur région (se pensant déracinés, se vivant expatriés, se définissant parfois explicitement tels), à rompre leur contrat de travail pour ainsi pouvoir revenir (pouvoir se « réenraciner »). En d'autres cas encore, les salariés perçoivent sans possible résolution leur éloignement. La distance (ou d'autres contraintes) interdit alors le schéma pendulaire entre « lieu » et « non-lieu » que certains adoptent (comme Stéphane). Le cas d'Anna illustre de manière exemplaire la tension existentielle alors vécue : installée depuis un an avec son compagnon à Rennes - suite à une affectation en tant que catégorie $\mathrm{B}$ dans la fonction publique, elle reconnaît de grands moments de solitude bien que l'adaptation au travail se passe, selon ses propres termes, " extrêmement bien ». Certes, elle se dit professionnellement entourée de personnes vivant la même situation de mobilité géographique - ce qui 
entraîne une certaine forme de solidarité entre collègues. Reste qu'elle se sent profondément « étrangère, au milieu des Bretons ». Marseille lui manque et, pour combler ce manque, elle quête tout ce qui est évocateur de son territoire - dans l'attente lancinante, de son retour - « chez elle». Anna dit ainsi se sentir « à deux endroits », en parlant de Marseille et de Rennes : «j'ai deux chez-moi, mais, là où j'habite, ce n'est pas vraiment chez moi... ». L'implication personnelle dans la région d'accueil, qui n'est définitivement pas «chez elle» est réduite - par « peur de s'investir », sachant le désir de partir. Autrement dit, la région d'accueil n'est pas ici vécue comme un «non-lieu»; elle n'est pas non plus un « lieu » véritable. Elle est un entre-deux, vécue parfois de manière ambigüe (tantôt comme « non-lieu », lors de moments d'accablement véritable, sombrant dans la nostalgie, tantôt comme « lieu ») et parfois de manière ambivalente (à la fois comme « lieu » et comme « non-lieu »).

À des degrés variés, cette nostalgie est très présente, dans tous les discours, plus souvent implicitement qu'explicitement. Certains en usent, pour aller mieux : elle peut effectivement aider à s'adapter aux difficultés vécues dans les mobilités (RobertDemontrond et Divard, 1998). Reste que la nostalgie est régulièrement déniée, fait symptomatique de la puissance normative de la figure du «nomade », du travailleur parfaitement mobile. Les propos tenus se révèlent ainsi souvent confus, lorsque le thème survient en cours d'entretien : « J'ai pas ce mal du pays comme les gens peuvent l'avoir à vraiment pas être pas bien, à ressentir le besoin... Si je rentre pas avant trois semaines (pause)... je suis jamais rentré, je suis jamais rentré... je suis jamais PAS ren- tré pendant un mois...» (Stéphane). Parfois, le terme générique est dénié (la faiblesse est atténuée, circonscrite à un cas particulier, localisé) : «J'ai pas le mal du pays, j'ai le mal de la région parisienne! Je me sentirais mieux en Bretagne dans ma région d'origine » (Enna). Parfois encore, le mal du pays vécu, lors de l'expatriation, transparaît (sans être dit véritablement) dans le récit du retour au pays : « Le jour où je suis parti, je savais que j'allais revenir. Lorsque j'ai aperçu le clocher de la cathédrale et croisé le panneau d'Autun à l'entrée de la ville, j'étais comme un gamin dans ma voiture. J'étais très ému. À Autun, je suis chez moi. » (Jocelin). On retrouve ici, typiquement, dans le cas de mobilités infranationales, les mêmes vécus que ceux rapportés dans les travaux à l'international concernant le «mobility syndrome ». Avec les mêmes conséquences d'importance, si la nostalgie n'est pas bien gérée. Plusieurs salariés rapportent ainsi leurs problèmes de déprime, ou même de dépression. Le cas de Marie est très illustratif du phénomène: «j'ai un gros gros manque de mon "pays". Spécifiquement du Finistère. J'ai l'impression que même si l'Aquitaine entre vignes et montagne, océan et ville est une région magnifique, rien n'égalera jamais mon département du Finistère. Alors quand comme ces derniers temps je ressens ce mal du pays (...) Je pense à l'océan, les jours de plage, le sentiment que $\mathrm{j}$ ' ai face à la mer qui se déchaîne. Je sais que ma nostalgie, ma mélancolie ne s'arrêtera jamais. Et pourtant je pense que ma vie est meilleure aujourd'hui. C'est dingue mais là-bas, c'est chez moi. J'aurais beau vivre cinquante ans ici, je suis sûre de ne jamais retrouver cette sensation. Le problème est que ma mère aussi avait ce sentiment, elle était partie suivre 
mon père à $50 \mathrm{~km}$ seulement, ils étaient revenus cinq ans plus tard après une tentative de suicide de ma mère. Ça fait sept ans que je suis ici, ça m'a manqué bien sûr, mais c'est depuis deux, trois mois que j'ai un manque viscéral, comme si on m'avait enlevé un organe... »

Outre le terme «d'expatrié », ceux « d'exilé » ou de « migrant » sont souvent utilisés par les salariés pour s'autodésigner, ou pour désigner le statut de ceux en situation de mobilité géographique, à l'infranational (parfois, d'ailleurs, à quelques kilomètres seulement de distance). Le malaise nostalgique s'observe ainsi jusqu'au plus près du «chez-soi ». Il induit, en stratégies d'adaptation, les mêmes comportements, les mêmes efforts de maintien d'une proximité physique et symbolique avec le pays que ceux observés dans les grandes migrations internationales. « À partir du moment où on part à quelques dizaines de $\mathrm{km}$, on peut ressentir le sentiment d'être déraciné. Le changement de pays n'est pas un facteur si déterminant. (...) On peut être déraciné à quelques dizaines de kilomètres ». Léo, de Bretagne, rapporte ainsi qu'il lui est « difficile de s'intégrer en Normandie», pour cause de trop grandes différences vécues dans l'art du vivre ensemble. Luc, parti de Bretagne à Laval, rapporte s'être aménagé un emploi du temps qui, chaque semaine (dans la logique pendulaire, oscillant entre «lieu» et «non-lieu»), lui permet « de franchir la "frontière" ".

En résumé, les salariés mobiles interrogés s'avèrent en constante tension entre deux territoires géographiques. Ils éprouvent de vives difficultés d'adaptation. Ils présentent, à l'infranational, les mêmes problèmes que ceux identifiés et étudiés à l'international - où l'échec se traduit parfois par un retour prématuré du salarié, coûteux pour les organisations. Si les conditions institutionnelles de la mobilité infranationale sont telles que ce retour anticipé est le plus souvent impossible, d'un point de vue intraorganisationnel, la rupture contractuelle avec l'organisation est parfois la solution retenue pour les individus. Individus pour qui la distinction entre mobilité choisie et mobilité subie n'a guère de sens.

\section{La complexité du processus identitaire}

Concernant les autres résultats saillants de notre étude, nous notons un lien fort entre mobilité et différences de compétence perçues (cf. sur ce point, Gherardi et Pierre, 2010) - différences parfois positives, parfois négatives, induisant des tensions vives entre mobiles et autres salariés de l'organisation. Plusieurs de nos informateurs rapportent être ou avoir été sujets à des moqueries : « Je ne me sens pas Normand et je me sens Lorrain, fier d'être Lorrain, fier d'avoir mon accent (...). Ah ben je me fais chambrer, je me fais chambrer, presque tous les jours hein de toute façon quand je discute avec des Normands, ils me connaissent pas, ils me disent 'toi, t'es pas Normand toi”. Donc heu... et fier de l'être hein, je fais "non, je suis un lorrain". (...) J'accentue la différence en fait... (...) Ouais je suis Lorrain, je suis Mosellan ! (Stéphane). De même qu'en mobilité internationale, des difficultés à communiquer dans une même langue apparaissent et peuvent poser des problèmes d'adaptation, de même à l'échelle infranationale, des différences linguistiques peuvent créer des tensions d'importance entre salariés "expatriés » et clients ou usagers " locaux » : «Alors là, j'ai vu des Alsaciens des vrais de vrai, qui quand tu, quand ils te parlent en alsacien, tu 
dis : je comprends pas... " ah ouais, mais vous venez d'où ?" “de Lorraine”... j'en ai eu plusieurs qui m'ont dit "vous me touchez pas"... le problème Alsace/Lorraine des patients... Après j'en ai eu: "mais vous venez travailler en Alsace, vous n'avez qu'à apprendre l'alsacien !" » (Tania). Ces tensions linguistiques, et d'autres liées aux pratiques culturelles, peuvent également survenir entre les salariés « expatriés » et leurs collègues « locaux »: «Parmi mes collègues (...) elles avaient trente, quarante ans, elles parlaient l'alsacien (entre elles), mais mieux que le français. (...) Et je me rappelle en décembre quand on me disait "tu ne vas pas au marché de Noël ?" je disais "c'est de la connerie ça", j'ai cru qu'elles allaient me tuer: "le marché de Noël ! Il faut y aller! Le vin chaud, il faut y goûter !" ". Si tu ne vas pas au marché de Noël, tu es foutu. C'est vraiment la tradition de la tradition » (Tania). Souvent, en réaction compensatoire à ces difficultés vécues, nos informateurs ont rapporté le désir de se retrouver entre personnes du même « territoire », du même « pays », et le plaisir pris en ces occasions. Dans le cadre du travail, ces rencontres biaisent significativement le cadre relationnel, l'écartant de la norme institutionnelle en enclenchant un régime de familiarité (d'« entre-soi »). « Mon premier patient, c'était en soins palliatifs, et c'était un Monsieur d'Oeting (...) Elle [sa femme] me dit “j'habite dans le 57 à Oeting (...)" Et là, avec ce monsieur-là, je me suis vraiment sentie à l'aise, je lui faisais sa toilette... en fait c'est comme si c'était mon papi. Il venait du même coin que moi, il fallait que je le chouchoute jusqu'à sa dernière minute, fallait vraiment qu'il soit à l'aise. Quand sa femme venait, je lui donnais tout, le café, la couverture, elle pouvait dormir la nuit et tout jusqu'à la dernière minute je ne l'ai pas lâché le papi » (Tania).

Parfois, le retour dans sa région, «chez soi », peut ne pas être d'évidence : l'expatrié peut de fait endosser l'image (subir le poids des stéréotypes) de « l'ailleurs » dont il provient, où il (n)'est (que) passé. «Le pire, c'est qu'ils vous considèrent désormais comme "le Parisien de service". » (Nicolas) - soit un sujet convaincu de ses compétences supérieures, dominateur, infériorisant, que l'on préjuge empli de préjugés, qui suscite la défiance... La désillusion est alors d'autant plus grande que le retour a été longtemps espéré, et ses conditions sublimées.

\section{III - LA TRAJECTOIRE DES SALARIÉS LORS D'UNE MOBILITÉ INFRANATIONALE : DISCUSSION ET PERSPECTIVES}

\section{Discussion}

\section{L'attachement au territoire chez les mobiles}

La recherche empirique entreprise, ethnosociologique, a permis d'élucider l'existence d'un attachement au territoire générant des tensions d'importance. Contre la doxa managériale, la mobilité géographique n'est pas d'évidence. Le concept de «capital de mobilité », développé par Murphy-Lejeune (2003), comme un ensemble de compétences évolutives, susceptibles de faciliter l'adaptation des individus en territoire étranger, s'impose à l'échelle infranationale.

Le « retour au pays » s'avère très diversement investi, intellectuellement et émotionnellement : tantôt demandé, espéré, attendu, voulu, tantôt estimé possible sous conditions, ou encore impossible. La démarche suivie permet de dépasser la distinction 
mobile/immobile - rapprochant nos observations de celles rapportées dans des travaux à l'international (cf. notamment Gustafson, 2009 ; Gherardi et Pierre, 2010) : le salarié « mobile» peut avoir des attaches, même quand la mobilité est voulue. Nos résultats questionnent les recherches antérieures sur la distinction mobilité voulue/mobilité subie (Vignal, 2005) qui n'a aucun sens pour les salariés et ils permettent de relativiser l'opposition qui est faite entre d'une part, le succès des «nomades » et d'autre part, l'échec des « enracinés». En effet, les entretiens auprès des salariés, répétés sur une longue période, permettent de comprendre l'évolution des motivations et surtout comprendre le tiraillement auquel doit faire face le salarié. Nous avons pu voir en particulier, toute la complexité des liens entre territoire et l'attachement $\mathrm{au}(\mathrm{x})$ lieu(x), pouvant créer des difficultés aux salariés volontaires qui n'ont pas subi de mobilité (celle-ci est d'ailleurs toujours à nuancer par rapport au poids que peut peser la norme sociale dans le choix de mobilité).

\section{Les difficultés rencontrées par les salariés en infranational}

Des comportements occultés dans la littérature ont été mis en évidence. Certains salariés vont jusqu'à démissionner pour refuser une mobilité imposée et pour retourner vers leur terre d'attaches, d'autres trouvent des solutions (se mettre à mi-temps annualisé) afin de privilégier leur vie privée. L'enquête ethnosociologique effectuée retrouve ainsi les travaux d'Alter (2011) et les complète : la logique du don (déracinement) et du contre don (retour au pays) n'est pas automatique, loin de là. L'entreprise semble tendanciellement oublier que le salarié peut être lié, émotionnellement et physiquement, à un territoire. L'engagement tacite du « retour au pays » systématique est ainsi banni (Alter, 2011), ou encore, l'entreprise laisse souvent espérer au salarié que sa demande de mobilité sera acceptée, jusqu'à l'épuisement de la situation. Dans les représentations des salariés, le «grand» n'est plus celui qui est mobile, contrairement à la définition de Boltanski et Chiapello (1999), mais celui qui arrive à rester « au pays » ${ }^{1}$. Enfin, des comportements nostalgiques sont identifiés, qui peuvent à la fois aider à l'adaptation, mais aussi mener à la dépression, si le retour dans la région d'origine ne se fait pas. Positivement vécue, la nostalgie favorise la mobilité ; négativement vécue, le ressenti douloureux de l'absence prévaut, devient pathologique. La nostalgie est ainsi tantôt adaptative, tantôt désadaptative : des perspectives de recherche sont à mener pour approfondir les dimensions de cette variable, trop peu étudiée encore (quels sont précisément ses antécédents ? Ses conséquences? Quelles sont les conditions du passage d'une nostalgie bénéfique à une nostalgie pathologique ?)

En définitive, nous notons la nécessité d'une rupture avec les préjugés courants, en gestion, sur la mobilité géographique: notamment celui qui consiste à croire que la mobilité géographique nationale est plus facile à gérer (pour le salarié, pour l'entre-

1. Selon Boltanski et Chiapello (1999), dans la cité par projets, le « grand » est celui qui est mobile et sans attache, car cette situation lui ouvre les portes de la réussite professionnelle. Est considéré comme " petit» au contraire, celui qui est immobile, car il se met ainsi en situation de précarité. Son ancrage et ses attaches ne lui permettent pas d'ouvrir son réseau et de rencontrer d'autres personnes. 
prise) que la mobilité internationale, parce qu'il n'y a pas de problème de différence culturelle. Les pratiques RH s'avèrent sousestimer l'importance de l'attachement territorial des salariés. Le principe qu'elles retiennent - «sortir des pratiques favorisant l'attachement à un métier, à un lieu » (Alter, 2011) - se révèle violent pour les salariés, et par cela même dysfonctionnel pour l'entreprise.

\section{Implications managériales et perspectives de recherches}

Nous avons détecté : i) les mêmes problèmes que ceux déjà identifiés en mobilité internationale (retour prématuré notamment) ; mais aussi ii) des situations beaucoup plus compliquées à gérer (notamment, l'identité de « salarié en déplacement »). Le traitement des échecs actuellement proposé en mobilité internationale, qui est répliqué à l'échelle nationale, n'est pas satisfaisant du fait du paradigme cognitiviste, behavioriste qui les inspire : les programmes de formation, d'une part, les aides matérielles et financières, d'autre part (traduction du « soutien à la famille»), ne suffisent pas. Quelques travaux à l'international mettent en avant une autre solution : aider le salarié à " développer des attentes plus réalistes » (Glanz et al., 2001). Dans cette perspective, les tensions identifiées incitent à étudier plus précisément les comportements nostalgiques positifs et négatifs. Des recherches antérieures ont déjà soulevé le lien pertinent entre le stress et la relocalisation des salariés, en identifiant le « mobility syndrome » (Stroh, 1999). Ce syndrome mis en avant n'a pas été approfondi et se traduirait par : « depression, (...) a low level of community involvement, a strong dependence on their marriage for emotional satisfaction, a signi- ficant rate of alcohol abuse, notable destructive aggression (...)» (Marshall et Cooper, 1979, cité par Stroh, 1999). Aucune étude en GRH n'a encore exploré ce concept en lien avec les politiques de mobilités des salariés. Le développement d'échelles de mesure, afin d'évaluer l'enracinement ou la nostalgie, ou encore l'attachement au territoire des salariés, reste une voie à privilégier.

La recherche empirique entreprise soulève diverses questions en termes de politiques RH et marketing et plus largement de gestion du territoire et des politiques régionales. Concernant la mobilité en France, certaines régions s'avèrent significativement moins attractives que d'autres. Pour pallier ce problème, des investissements conséquents sont engagés dans le marketing des territoires (par exemple ceux développés par l'Auvergne pour accroître son attractivité démographique). Si la solution paraît séduisante, de récents travaux en marketing sur le tourisme à l'international pointent cependant l'existence de risques. En effet, la destination est alors idéalisée, présentée en des termes pouvant être déceptifs. Les problèmes d'adaptation peuvent être accrus.

\section{CONCLUSION}

Nous avons élucidé le lien entre la mobilité géographique, l'attachement au territoire et la souffrance des salariés en situation de mobilité infranationale, en associant l'expérience et le ressenti des salariés. L'articulation entre la méthodologie et la théorie est ici d'importance : une recherche qui nécessite, nous l'avons vu, de donner la parole aux principaux concernés, les salariés. Nous avons ainsi pu montrer que l'exploitation des modèles existants dans la littérature 
et leur réplication paraissent saturer l'univers des possibles concernant la compréhension des échecs à la mobilité et la proposition de nouvelles solutions sur le plan opérationnel. En effet, le traitement des échecs n'est pas satisfaisant, puisqu'il soulève un problème d'adaptation; celle-ci étant envisagée comme instrumentale, fonctionnelle. En conséquence de quoi, une des solutions proposées au salarié - essentiellement dirigée vers un apprentissage cognitif - est d'acquérir des compétences afin de faire face à l'impératif économique, à l'injonction d'être mobile en tout lieu, à tout moment. Un changement de paradigme est donc pertinent. Un nouveau cadre théorique doit s'inspirer d'autres disciplines scientifiques, et notamment, l'émergence du phénomène de nostalgie reste à explorer.
An tant que syndrome d'adaptation, la nostalgie ne s'oppose pas au changement, mais au contraire, si elle est identifiée et gérée, elle peut aider le salarié à s'adapter. $\mathrm{Au}$ regard des résultats, de nouvelles perspectives en GRH semblent plaider pour une prise en compte du territoire, de l'environnement - au sens large - et des liens d'attaches du salarié avec cet environnement. La construction identitaire du salarié ne se situe pas seulement sur le plan de l'identité professionnelle, elle intervient également lorsque le salarié est confronté à différents milieux, territoires. L'orientation actuelle de la GRH face aux problèmes d'adaptation lors d'une mobilité géographique, en se concentrant sur les fragilités individuelles et familiales, contribue à occulter, nier les liens entre le salarié et son ancrage au sol.

\section{BiBLIOGRAPHIE}

Alter N. (2011). « Don, ingratitude et management. Suicide et désengagement au travail », Revue française de gestion, vol. 2, $\mathrm{n}^{\circ} 211$, p. 47-61.

Augé M. (1992). Non-lieux, introduction à une anthropologie de la surmodernité, Seuil, Paris.

Bertaux D. (2005). L'enquête et ses méthodes : le récit de vie, $2^{\mathrm{e}}$ édition, Armand Colin, Paris.

Boltanski L., Chiapello E. (1999). Le nouvel esprit du capitalisme, Gallimard, Paris.

Di Méo G. (1998). Géographie sociale et territoires, Nathan, Paris.

Gherardi L., Pierre P. (2010). « Mobilités géographiques et écarts de pouvoir au sein de trois entreprises mondialisées. Mobiles, immobiles et "ubiquistes" ", Revue européenne des migrations internationales, vol. 26, $\mathrm{n}^{\circ} 1$, p. 161-185.

Glanz L., Williams R., Hoeksema L. (2001). "Sensemaking in expatriation, a theoretical basis", Thunderbird International Business Review, January-February, p. 101-119.

Guillot-Soulez C., Sergot B. (2010). «Qui m'aime me suive ! : la mobilité des salariés à l'épreuve des transferts d'établissements », Revue Management \& Avenir, $\mathrm{n}^{\circ} 33$, p. 72-94. Gustafson P. (2009). "Mobility and territorial belonging", Environment and Behavior, vol. 41, n 4, july, p. 490-508.

Hine C. (2000). Virtual Ethnography, Sage, London. 
Juan S. (2005). « La "socioanthropologie" : champ, paradigme ou discipline ? », Bulletin de méthodologie sociologique, 87, p. 61-79.

Lauriol J., Perret V., Tannery F. (2008). « Stratégies, espaces et territoires : Une introduction sous un prisme géographique », Revue française de gestion, vol. 34, n 184, p. 91-103.

Lemoine M., Wasmer E. (2010). Les mobilités des salariés, Rapport du Conseil d'analyse économique (CAE), n 90, p. 3-87.

Mignonac K. (2001). "Les déterminants de la disposition envers la mobilité intraorganisationnelle: étude auprès d'une population d'ingénieurs », M@nagement, vol. $4, \mathrm{n}^{\circ} 2$, p. 47-78.

Murphy-Lejeune E. (2003). L'étudiant européen voyageur, un nouvel étranger, Didier, Paris.

Oberg K. (1960). "Culture shock: adjustment to new cultural environment", Practical Anthropologist, 7, p. 177-182.

Paillé P., Mucchielli A. (2008). L'analyse qualitative en sciences humaines et sociales, Armand Colin, Paris.

Ravelli Q. (2008). «Cadres, techniciens et ouvriers : mobilités professionnelles et privilège spatial », Espaces et sociétés, vol. 4, n 135, p. 157-171.

Ricœur P. (1990). Soi-même comme un autre, Seuil, Paris.

Robert-Demontrond P. (2001). «Psychodynamique de l'expatriation. La nostalgie comme syndrome d'adaptation », Revue Internationale de Psychosociologie, ${ }^{\circ} 16-17$, p. 317-338.

Robert-Demontrond P., Divard R. (1998). «La nostalgie comme symptôme de rejet de l'autonomie au travail: perspectives étiologique, sémiologique et ingénierique », Le Travail Humain, vol. 61, n 3, p. 235-262.

Roques O., Roger A. (2004). « La gestion de la mobilité géographique des salariés : le cas de Technicatome », Revue française de gestion, vol. 30, $\mathrm{n}^{\circ} 150$, p. 71-85.

Stroh L.-K. (1999). "Does relocation still benefit corporations and employees ? An overview of the literature», Human Resource Management Review, vol. 9, n 3, p. 279-308.

Vignal C. (2005). «Injonctions à la mobilité, arbitrages résidentiels et délocalisation de l'emploi », Cahiers internationaux de sociologie, vol. 1, n 118 , p.101-117. 